Original Article

\title{
Epigenetic dysregulation of genes related to synaptic long-term depression among adolescents with depressive disorder and sleep symptoms
}

\author{
Antti-Jussi Ämmälä a, b, *,1, Anna-Sofia Urrila b, c, 1, Aleksandra Lahtinen a, d, \\ Olena Santangeli ${ }^{\mathrm{d}}$, Antti Hakkarainen ${ }^{\mathrm{e}}$, Katri Kantojärvi ${ }^{\mathrm{a}}{ }^{\mathrm{b}}$, Anu E. Castaneda ${ }^{\mathrm{f}}$, \\ Nina Lundbom ${ }^{\mathrm{e}}$, Mauri Marttunen ${ }^{\mathrm{b}, \mathrm{c}}$, Tiina Paunio ${ }^{\mathrm{a}, \mathrm{b}}$ \\ a Department of Genetics and Biomarkers, National Institute for Health and Welfare, Helsinki, Finland \\ ${ }^{\mathrm{b}}$ Department of Psychiatry, University of Helsinki and Helsinki University Hospital, Helsinki, Finland \\ ${ }^{c}$ Department of Health, Unit of Mental Health, National Institute for Health and Welfare, Helsinki, Finland \\ d Department of Physiology, Faculty of Medicine, University of Helsinki, Helsinki, Finland \\ e Helsinki Medical Imaging Center, Radiology, University of Helsinki and Helsinki University Hospital, Helsinki, Finland \\ ${ }^{\mathrm{f}}$ Department of Welfare, Equality and Inclusion Unit, National Institute for Health and Welfare, Helsinki, Finland
}

\section{A R T I C L E I N F O}

\section{Article history:}

Received 10 August 2018

Received in revised form

18 January 2019

Accepted 23 January 2019

Available online $\mathrm{xxx}$

\section{Keywords:}

Adolescence

Depression

Epigenetics

Mood

Polysomnography

Sleepiness

\begin{abstract}
A B S T R A C T
Objectives: This study aimed to test the hypothesis that sleep and depression have independent effects on brain development and plasticity in adolescents, and that these changes are reflected in changes in the epigenome.

Methods: Participants were 17 medication-free adolescent boys (age $16.05 \pm 0.80$ years, mean \pm standard deviation (SD); eight cases with depression and sleep symptoms, nine healthy controls). Sleep was assessed by polysomnography recordings and the Pediatric Daytime Sleepiness Scale (PDSS) and Athens Insomnia Scale (AIS). Participants underwent a clinical evaluation. DNA methylation of blood leukocytes was measured by Illumina 450K array, and Ingenuity Pathway analysis was applied to identify the most significant pathways with differentially methylated positions (DMPs). Secondary analysis of the identified loci included linear correlations between methylation and the subjectively rated scales of sleep, depression and sleep microarchitecture.

Results: Due to small sample size, we found no genome-wide significant differences in methylation between cases and controls. However, pathway analysis identified the synaptic long-term depression (LTD) canonical pathway ( $\mathrm{p}=0.00045$ ) when the best 500 DMPs from the original case-control design were included. A flattened dissipation of slow wave sleep, tiredness and depression severity values correlated with five of 10 sites from the LTD pathway (IGF1R, PLAG16, PLA2R1, PPP2C5 and ERK12) in the secondary analysis when the case-control status was controlled for.

Conclusion: Among adolescents, depressive disorder with sleep symptoms is associated with a distinctive epigenetic pattern of DNA methylation in blood leukocytes. The enrichment of DMPs on genes related to synaptic LTD emphasizes the role of sleep in synaptic plasticity and the widespread physiological consequences of disturbed sleep.
\end{abstract}

(c) 2019 Elsevier B.V. All rights reserved.
* Corresponding author. National Institute for Health and Welfare, Mannerheimintie 166, P.O. 30, 00271 Helsinki, Finland.

E-mail address: antti-jussi.ammala@thl.fi (A.-J. Ämmälä).

1 These authors contributed equally to this work.

\section{Introduction}

Adolescence is characterized by major changes in sleep patterns. Despite being a period of rapid increases in physical and mental capabilities, it is paradoxically also a period of augmented vulnerability to psychiatric and sleep disorders, and the incidence of both insomnia and major depression increases simultaneously during adolescence [1]. 
Symptomatology in insomnia and depression are tightly linked and share many common features as well as common mechanisms $[2,3]$. Disordered sleep also increases the risk of depression [4,5]. The majority of patients with depressive disorder have symptoms of disturbed sleep (insomnia or hypersomnia), which also belong to the diagnostic criteria of the disorder [6]. Further, disturbances in cognitive functions are common to both conditions. A diminished ability to think or concentrate are hallmarks of clinical depression [6], while sleep deprivation and chronic sleep disturbance lead to reduced attention and vigilance, as well as impairments in memory and learning. Information acquisition takes place during waking, most effectively during high vigilance, while sleep promotes memory consolidation. The cellular mechanisms for memory formation comprise the activity-dependent plasticity processes of long-term potentiation (LTP) and long-term depression (LTD). They involve a number of biological processes ranging from changes in gene expression to synthesis of new proteins and comprising hundreds of molecules and molecular pathways [7]. Sleep deprivation, on the other hand, may impact structural plasticity via synaptic downscaling or by reducing spine density and attenuating synaptic efficacy in the hippocampus [8], which might be one of the cellular mechanisms linking depression, disturbed sleep and cognitive functions $[9,10]$.

Similarities between depression and disturbed sleep extend beyond signs and symptoms from the brain. Both conditions cooccur with low-level systemic inflammatory reactions and corresponding changes in gene expression in cells of the immune system, including blood leukocytes [11,12]. They are also frequently comorbid with other medical conditions associated with systemic inflammation, such as obesity or metabolic syndrome [13,14]. Thus, despite a neural origin, depression and insufficient sleep can be considered systemic disorders affecting functions of the whole body.

Insomnia and depression are both frequently triggered by environmental stressors, such as psychological stress or trauma [15-17]. DNA methylation is an epigenetic mechanism that allows interaction between gene expression and the environment. The role of the epigenetic regulation of sleep and emotions is emerging, and changes in DNA methylation have been linked to different forms of depression as well as to antidepressant response [18]. Furthermore, altered expression of histone deacetylases, major methylating enzymes, was found in depressive states, but not after remission, suggesting that methylation-driven changes in gene expression can play a role in the pathophysiology of depression [19]. In mice, sleep deprivation causes marked alterations in DNA methylation in the cortex, especially in the genes involved in synaptic plasticity, and concordant RNA expression changes [20]. These methylation changes are possibly driven by LTP, a major component in synaptic plasticity [21]. Additionally, in young healthy men, total sleep deprivation caused marked changes in the methylation of white blood cells and RNA expression [20]. In a large cohort of older women, insomnia symptoms, but not shortened sleep, were associated with altered methylation of peripheral blood leukocytes [22].

Given the systemic physiological effects of sleep insufficiency and depression, we hypothesized that sleep difficulties and depression have an effect on DNA methylation in peripheral blood leukocytes. To our knowledge, no studies in human clinical samples exist concerning the effect of sleep disturbances and depression on DNA methylation. We studied this in a sample of depressed adolescents with sleep difficulties compared to age- and gendermatched controls. We were particularly interested in identifying deviating patterns of DNA methylation in genes from pathways related to disturbed sleep. Finally, given the impact of sleep difficulties on depression in the short and long term, we hypothesized that sleep difficulties could show an effect on DNA methylation that is not entirely explained by depression severity.

\section{Methods}

\subsection{Subjects}

A total of 20 non-medicated adolescent boys aged 14.70-17.40 years (mean 16.05 years) participated in this study. Ten were cases all suffering from depressive and sleep symptoms recruited from the Helsinki University Central Hospital Department of Adolescent Psychiatry outpatient units, while 10 were healthy controls recruited via advertisements in a newspaper for the hospital staff. Due to dropout, genetic blood samples were not available for two subjects in the case group and one subject in the control group, leaving a total of 17 subjects in the analyses presented in this paper. The adolescents took part in a larger research project focusing on adolescent depression, sleep and brain maturation (the ADSLEEP project) [23-25]. Both the subjects and their parents or legal guardians gave written informed consent for participating in the study, and the study protocol (Fig. 1) was approved by the ethics committee of the Helsinki University Central Hospital.

Exclusion criteria for all subjects included mental retardation, chronic somatic illness, current use of medication, substance abuse/ dependence, age over 17.5 or under 14.5 years, insufficient

$\begin{array}{lllll} & \text { Outpatient/ } & \text { Hospital } & \text { Adolescent's home } & \text { Outpatient/research unit } \\ \text { Location } & \text { research unit } & \text { laboratory } & 2 \text { consecutive } & 1 \text { visit directly after PSG } \\ & 1-2 \text { visits } & 1 \text { visit } & \text { nights }\end{array}$

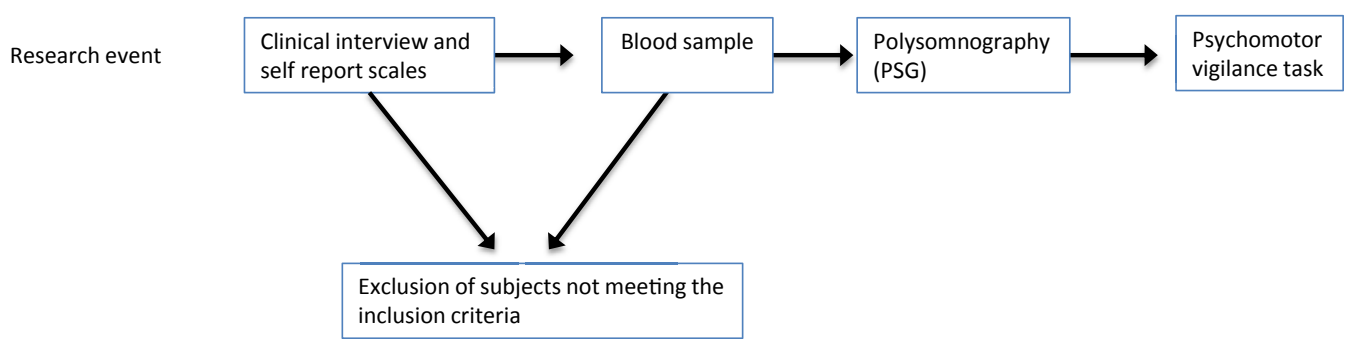

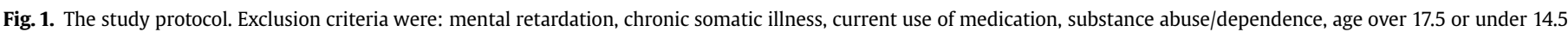

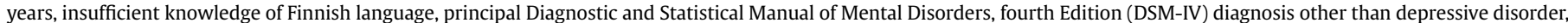

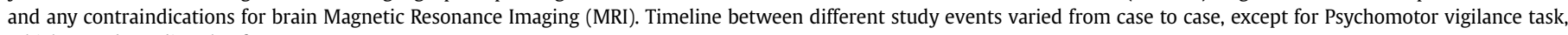
which was done directly after PSG. 
knowledge of Finnish language, principal Diagnostic and Statistical Manual of Mental Disorders, fourth Edition (DSM-IV) diagnosis other than depressive disorder, and any contraindications for brain magnetic resonance imaging (MRI). All subjects remained free of psychotropic and other medications during the entire study period. The presence of somatic conditions was ruled out based on blood samples, which included measurements of blood cell count, serum testosterone, serum luteinizing hormone, C-reactive protein, fasted blood sugar levels, and liver, kidney and thyroid function measurements. No structural pathologies were found in their brain anatomy according to brain MRI evaluated by a neuroradiologist. The subgroups of cases and controls did not differ in terms of their age, body mass index (BMI), or serum testosterone levels (independent samples $t$-tests $p>0.05$; Table 1 ). All subjects consumed less than three cups of coffee daily (or the equivalent amount of other caffeinated products). Subject characteristics are described in detail in Supplementary Table S1.

\subsection{Psychiatric evaluation}

The present and lifetime episodes of DSM-IV axis I disorders were assessed with a semi-structured diagnostic interview, the Schedule for Affective Disorders and Schizophrenia for School-Age Children: Present and Lifetime version (K-SADS-PL) [26]. The same clinician (A.S.U.) performed all of the interviews, and the diagnoses were confirmed in a diagnostic meeting with a senior clinician (M.M.). No axis I diagnoses were found among the controls. The cases were confirmed to suffer from depressive disorder (lifetime first depressive episode for six, second episode for one of the cases; mean length of the current depressive episode at the time of interview, $58 \pm 53$ weeks), except for one case who only suffered from a circadian rhythm sleep disorder with minor mood symptoms (pessimistic views of the future, feelings of failure, disappointment in oneself, self-criticism, fatigue). None of the cases manifested psychotic features or suffered from bipolar disorder.
The majority of cases (6/8) did not have comorbid axis I disorders. Comorbid anxiety disorder was present in one case and comorbid disruptive behaviour disorder in another.

As part of the DSM-IV axial diagnostic procedure, the global assessment of functioning scale (GAF; numeric range of 0-100) was used according to DSM-IV guidelines to assess overall psychosocial functioning [6]. GAF has previously been used in studies among adolescents $[27,28]$.

Depression symptom severity was assessed with the 21-item Beck Depression Inventory (BDI-21), a standardized 21-item selfrating questionnaire [29] which has been well studied in adolescents $[30,31]$. In the BDI-21, subjects are asked to rate each of the symptoms on a four-point scale (range 0-3) according to the severity of the symptom (sum score range 0-63). In correlation analyses with differentially methylated positions (DMPs), a shorter version of the BDI, excluding the sleep and tiredness items, annotated here as BDI-19 (sum score range 0-57), was applied.

\subsection{Psychomotor vigilance task}

The psychomotor vigilance task (PVT) is a widely used neurobehavioural test to assess sustained attention [32]. As part of a larger neuropsychological test battery, a 5-min version of the PVT task was performed with a PVT-192 unit (Ambulatory Monitoring, Inc., Ardsley, NY, USA). During the task, a digital millisecond counter starts to scroll on a small computer screen at random intervals of $2-10 \mathrm{~s}$, and the subjects have to respond by pressing a button as quickly as possible to stop the counter. After each press, immediate feedback on performance is displayed on the screen.

The tests that differed between cases and controls $(p<0.05$, one-way analysis of variance (ANOVA)) were median reaction time (Median RT) $(p=0.022)$, minimum reaction time (MIN RT) $(p=0.007)$ and fastest $10 \%$ mean reaction time (FRT) $(p=0.010)$. We chose Median RT to represent vigilance in the secondary analysis.

Table 1

Sample characteristics presented for the total sample $(N=17)$, and separately for the cases $(N=8)$ and controls $(N=9)$.

\begin{tabular}{|c|c|c|c|c|}
\hline Variable & & Mean & SD & Range \\
\hline \multirow[t]{3}{*}{ Age $(N=17)$} & & 16.05 & 0.80 & $14.70-17.40$ \\
\hline & Cases $(N=8)$ & 15.93 & 0.86 & $14.70-17.30$ \\
\hline & Controls $(N=9)$ & 16.16 & 0.78 & $14.80-17.40$ \\
\hline \multirow[t]{3}{*}{$\operatorname{BMI}(N=17)$} & & 21.22 & 2.77 & $16.78-27.55$ \\
\hline & Cases $(N=8)$ & 21.89 & 3.58 & $16.78-27.55$ \\
\hline & Controls $(N=9)$ & 20.62 & 1.83 & $17.82-23.80$ \\
\hline \multirow[t]{3}{*}{ S-Testo $(N=17)$} & & 19.79 & 3.77 & $15.00-27.60$ \\
\hline & Cases $(N=8)$ & 20.28 & 4.12 & $15.00-26.30$ \\
\hline & Controls $(N=9)$ & 19.36 & 3.63 & $15.30-27.60$ \\
\hline \multirow[t]{3}{*}{ BDI-21 $(N=17)$} & & 9.65 & 11.05 & $0-33$ \\
\hline & Cases $(N=8)$ & $17.63^{\mathrm{a}}$ & 11.05 & $6-33$ \\
\hline & Controls $(N=9)$ & $2.56^{\mathrm{a}}$ & 4.16 & $0-12$ \\
\hline \multirow{3}{*}{$\operatorname{GAF}(N=17)$} & & 66.76 & 16.57 & 43-90 \\
\hline & Cases $(N=8)$ & $50.62^{\mathrm{b}}$ & 6.16 & $43-59$ \\
\hline & Controls $(N=9)$ & $81.11^{\mathrm{b}}$ & 4.86 & $75-90$ \\
\hline \multirow[t]{3}{*}{ AIS $(N=17)$} & & 6.12 & 6.11 & 0-18 \\
\hline & Cases $(N=8)$ & $10.75^{\mathrm{b}}$ & 5.75 & $1-18$ \\
\hline & Controls $(N=9)$ & $2.00^{\mathrm{b}}$ & 2.29 & $0-7$ \\
\hline \multirow[t]{3}{*}{$\operatorname{PDSS}(N=16)$} & & 15.94 & 6.07 & $6-26$ \\
\hline & Cases $(N=7)$ & $19.71^{\mathrm{a}}$ & 5.35 & $12-26$ \\
\hline & Controls $(N=9)$ & $13.00^{\mathrm{a}}$ & 5.05 & $6-21$ \\
\hline \multirow[t]{3}{*}{$\operatorname{TST}(N=17)$} & & 454.35 & 69.10 & $312.5-529.5$ \\
\hline & Cases $(N=8)$ & $412.31^{\mathrm{a}}$ & 82.22 & $312.5-517.0$ \\
\hline & Controls $(N=9)$ & $491.72^{\mathrm{a}}$ & 17.21 & $469.0-529.5$ \\
\hline
\end{tabular}

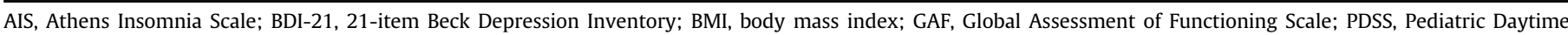
Sleepiness Scale; S-Testo, serum testosterone level (nmol/L); SD, standard deviation; TST, total sleep time (min). In bold both groups combined.

a Statistically significant differences between the cases and controls at a statistical threshold of $p<0.05$.

b Statistically significant differences between the cases and controls at a threshold of $p<0.001$ (independent samples $t$-tests). 


\subsection{Assessment of sleep}

The sleep symptoms were assessed as part of the K-SADS-PL interview attachment for affective disorders, which contains six items on sleep (initial insomnia, middle insomnia, terminal insomnia, sleep-wake rhythm disturbance, non-restorative sleep, and hypersomnia). The interviewer rates each symptom as nonexistent, sub-threshold, or clinically significant according to standard criteria, explained in detail previously [33]. The patients' clinically significant sleep symptoms are listed in Supplementary Table S1. No clinically significant sleep symptoms were found among the controls.

The Athens Insomnia Scale (AIS) was used to assess insomnia symptoms during the past month based on International Classification of Diseases 10th edition (ICD-10) criteria [34]. The AIS is a validated self-report questionnaire consisting of eight items rated on a scale of $0-3$ (total sum score range $0-24$ ) on sleep induction, awakenings during the night, final awakening, total sleep duration, sleep quality, daytime well-being, daytime functioning capacity and sleepiness during the day.

The Pediatric Daytime Sleepiness Scale (PDSS) was used to measure daytime sleepiness [35]. The PDSS is a self-rated sleepiness scale specially designed for use among school-aged adolescents. It consists of eight items rated on a scale of $0-4$ (total sum score range $0-32$ ) related to daytime alertness, daytime tiredness/ grumpiness, trouble waking up, frequency of falling asleep/ drowsiness in class and while doing homework, falling back to sleep after being awakened, need for outside help to wake up and thoughts of needing more sleep.

Sleep architecture was measured with polysomnography including electroencephalogram (EEG), electrooculogram (EOG) and chin electromyogram (EMG) recordings. Polysomnography was performed in the adolescents' home environment using portable recording devices (Embla, Flaga Hf. Medical devices, Reykjavik, Iceland; EEG derivations F4-M1, C4-M1, O2-M1 and backup derivations F3-M2, C3-M2, and O1-M2; sampling rate $200 \mathrm{~Hz}$ ). The electrode positions were determined according to the International 10-20 system. Polysomnography was performed for two consecutive nights and, for the purposes of this study, the first night (adaptation night) was discarded and the second night of polysomnography was used in the analyses. One of the most important functional parameters of the sleeping EEG, slow wave sleep (SWA), was calculated using a power spectral analysis according to a previously described procedure [23]. Because in previous work with the same sample of adolescents we detected a flattening of SWA dissipation associated with depression especially in the frontal electrodes [23], we also wanted to explore its effect on methylation in this study.

\subsection{Epigenetic analyses}

Blood samples were obtained from peripheral venous sampling and DNA was extracted according to standard laboratory methods. The blood samples were taken at varying intervals from the clinical measurements (average time between clinical interview and blood sampling was 25 days \pm 51 ; range 3-218 days), but since the same investigator also conducted the post-sampling procedures (A.S.U.) and there were no apparent changes in the clinical status of the cases and controls, we can assume that they were symptomatic or asymptomatic, respectively. For depressed cases, the average time between the beginning of depression episode and blood sampling was 415 days (range 78-1078 days; standard deviation (SD) \pm 377 days). We therefore explored the association between time spent depressed and level of methylation for those loci of special interest.

\subsection{Illumina $450 \mathrm{~K}$ workflow}

DNA was bisulfide converted with the EZ-methylation kit (ZYMO research, Irvine, CA, USA). Bisulfite-converted DNA was then analysed with the Illumina Infinium HumanMethylation450 BeadChip Kit (Illumina Inc., San Diego, CA, USA) at the Estonian Genome Center. Quality control (QC) was performed using R software $(R \geq 3.0 .1)$ with specific algorithms implemented in $R$ packages 'minfi', 'IlluminaHumanMethylation450kmanifest' and 'limma' [36]. At this stage, probes with a detection of $p>0.01$ for any DNA sample or those missing measurements, known as crossreactive probes, probes on $\mathrm{X}$ and $\mathrm{Y}$ chromosomes, and probes that contained either a single nucleotide polymorphism (SNP) at the $\mathrm{CpG}$ interrogation site or at the single nucleotide extension were excluded from subsequent analysis. Altogether, 46,384 probes were excluded, leaving 439,128 probes for subsequent analysis. Normalization of the $450 \mathrm{~K}$ data was performed using the subsetquantile within-array normalization (SWAN) function in R-package 'minfi', which takes into account the fact that the Illumina $450 \mathrm{k}$ chip uses two types of assays [37,38] with two different types of chemistry, making different probes comparable to each other. We calculated estimates for white blood cell populations of each sample with 'limma' and compared estimated populations for cases and controls with $t$-tests (Supplementary Table S2) [39,40]. No significant differences $(p<0.05)$ were found in cell population distributions between cases and controls. All raw values in $450 \mathrm{~K}$ were then converted to M-values in order to take into account the non-linear Bernoulli-type distribution of the raw values in the array, which creates biased methylation values, especially at highly methylated probes [41]. The M-value is calculated as the $\log _{2}$ ratio of the intensities of a methylated probe versus unmethylated probe, and describes a measurement of how much more a probe is methylated than unmethylated. A positive $\mathrm{M}$-value, hence, means that more than half of a probe is methylated. M-values were then used in data analysis.

\section{Statistical analyses}

\subsection{Genome-wide analysis of DNA methylation in cases compared to controls}

In genome-wide analysis of the data from the Illumina $450 \mathrm{~K}$ chip, we used an empirical Bayes moderated $t$-test in probe-wise comparisons to compare the methylation values between cases and controls and to thereby identify DMPs. This approach was used because it is especially well-suited for experiments with a small number of arrays and for both one- and two-channel microarray experiments, such as the Illumina 450K [42]. To correct for multiple testing, we used the Benjamini-Hochberg procedure $(\mathrm{BH})$ in Rpackage 'limma' [43], which is based on false discovery rate (FDR) calculations using a $p<0.05$ cut-off. We set genome-wide significance to $5.0 \times 10^{-8}$ [36].

\subsection{Pathway analysis}

The 500 sites that differed the most between cases and controls according to the genome-wide analysis were entered into Ingenuity Pathway Analysis (IPA; Ingenuity Systems, Redwood City, CA, USA) to assess the biological relevance of dysregulated DMPs in cases compared to controls. This threshold was chosen to yield a manageable number of sites for pathway analysis. After removing sites that did not pass the QC procedure described above, there were 486 sites remaining. Of those sites, IPA could annotate 332 to a known gene in its pathway repository. A list of those genes is shown in Supplementary Table S3. IPA compares a given set of genes to 
previously known biological pathways and uses a right-tailed Fisher's exact test to evaluate whether this gene set is enriched to some pathway more than would be expected by chance. It does not correct for multiple testing, but correction for multiple testing would only affect the magnitude of a detected $p$-value, not which pathways arise from the analysis or the order of pathways.

Our top canonical pathway was the synaptic LTD pathway ( $p=0.00045$ ) followed by the Nitric Oxide Signaling pathway (NOS) signalling in skeletal muscle cells pathway and netrin signalling pathway both with nearly 10 -fold higher $p$-value $(p=0.0042)$. All $p$-values are nominal.

A canonical pathway refers to a molecular pathway that is an idealized or generalized pathway representing common properties of a module or pathway [44]. We chose to continue with canonical pathways as they are the most generalizable across species and tissues and are considered to be the most common pathways of a module or pathway.

\subsection{Correlation between methylation and depression, indices of sleep and neuropsychological performance}

We used linear regression between methylation and mood, tiredness and sleep, and neuropsychological measurements. Since original case-control status was expected to have an effect on results in these secondary analyses, we denoted whether the association was also significant after controlling for the case-control status by including the status as a covariate in the analysis. In addition, we denoted whether the association survived Bonferroni correction for multiple testing. All analyses were performed using SPSS v.24 (IBM, Armonk, NY, USA).

\section{Results}

\subsection{Epigenome-wide analysis between cases and controls}

We first compared the genome-wide DNA methylation levels of depressed adolescents with healthy controls using the Illumina $450 \mathrm{~K}$ array. There were no genome-wide significant differences in methylation levels between cases and controls (all $p>5 \times 10^{-8}$ ).

Next, we proceeded to pathway analysis by including 500 sites that differed most in cases vs controls upon initial analysis, comprising 332 sites that annotated to a known gene. Our top canonical pathway was the synaptic LTD pathway (10/165 sites) $(p=0.00045)$, followed by the NOS signalling in skeletal muscle cells pathway and Netrin signalling pathway, both with a nearly 10fold higher $p$-value $(p=0.0042)$.

Sites in the LTD pathway that were present in the top 500 DMPs were cg16841761 at Calcium Voltage-Gated Channel Auxiliary
Subunit Gamma 1 (CACNG1), cg22025854 at Calcium Voltage-Gated Channel Auxiliary Subunit Gamma 6 (CACNG6), cg08364956 at a heterochromatic area of Glutamate Receptor, Metabotropic 6 (GRM6), cg05110803 at a weak enhancer region of the Insulin Like Growth Factor 1 (IGF1R), cg19161850 at a promoter region of the gene encoding for Extracellular Signal-Regulated Kinase 1 and 2 (ERK12) (also known as Mitogen-Activated Protein Kinase 1, MAPK1), cg12066398 at a promoter region of Phospholipase A2 Group XVI (PLA2G16), cg04367351 at Phospholipase A2 Receptor 1 (PLA2R1), cg02263165 at a promoter region and downstream transcription start site of Protein Phosphatase 2 Regulatory Subunit 'B' gamma' (PPP2R5C), cg18823846 at Protein Kinase, CGMPDependent, Type I (PRKG1) and $\operatorname{cg} 25405123$ at a bivalent promoter region of Ryanodine Receptor 3 (RYR3).

Among these sites, DMPs at GRM6, CACNG1 and IGF1R showed lower, and all other sites higher, levels of methylation in cases compared to controls (relative hypo- and hypermethylation, respectively) (Table 2 ).

\subsection{Secondary analysis with three domains and methylation of sites related to the LTD pathway}

Given overall statistical evidence from the pathway analysis and previous evidence on the intimate connection between sleep, mood and cognition $[45,46]$, we focused the secondary analysis on the DMPs identified from the LTD pathway.

Secondary analyses utilized linear regression to model associations between the 10 methylation loci for the LTD pathway and the subjective and objective measures for depressed mood (BDI-19), tiredness (PDSS) and vigilance (median RT), and sleep (AIS, SWA dissipation) in the whole sample. Since only one site, cg12066398 (PLA2G16), showed correlation between methylation and depression length ( $\beta=0.860, p=0.013$ uncorrected for multiple testing), we decided not to adjust analyses by depression length. Adjusting would have abolished controls from the analysis, leaving the sample size very low (Table 3 ).

In linear regression models with no covariates, we found associations between mood (BDI-19) and PLA2G16 ( $\beta=0.77$, $p=0.00031)$ and with tiredness (PDSS) and IGF1R $(\beta=-0.79$, $p=0.00029)$, ERK $12(\beta=0.72, p=0.002)$ and PLA2R1 $(\beta=0.77$, $p=0.00047)$. We also found an association with sleep (AIS) and $R Y R 3$ ( $\beta=0.76, p=0.00041$ ). Finally, we found an association between PPP2R5C and SWA dissipation $(\beta=-0.67, p=0.004)$. Of these, results from PLA2G16, IGF1R, PLA2R1 and RYR3 survived Bonferroni correction (Table 3 ).

In linear regression models where original case-control status was taken into account as a covariate, we found several nominal associations between certain sites and parameters. Nominal

Table 2

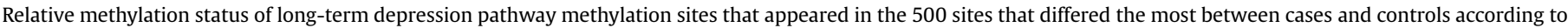
the genome-wide analysis their respective genes, and values in cases and controls.

\begin{tabular}{|c|c|c|c|c|}
\hline Locus & Gene & $p$-value in $450 \mathrm{~K}$ array & Methylation level in cases vs controls ${ }^{\mathrm{a}}$ & Average $\mathrm{M}$-value ${ }^{\mathrm{b}}$ for cases/controls \\
\hline $\operatorname{cg} 1684176$ & CACNG1 & 0.000381 & Lower & $3.18 / 3.49$ \\
\hline cg22025854 & CACNG6 & 0.000527 & Higher & $-1.17 /-1.57$ \\
\hline cg08364956 & GRM6 & 0.000838 & Lower & $3.29 / 3.56$ \\
\hline cg05110803 & $I G F 1 R$ & 0.000306 & Lower & $1.47 / 1.95$ \\
\hline $\operatorname{cg} 19161850$ & ERK12 & 0.000663 & Higher & $-3.88 /-4.13$ \\
\hline cg12066398 & PLA2G16 & 0.000540 & Higher & $-3.70 /-4.07$ \\
\hline cg04367351 & PLA2R1 & 0.000659 & Higher & $-4.14 /-4.46$ \\
\hline cg02263165 & PPP2R5C & 0.000207 & Higher & $-3.14 /-3.35$ \\
\hline $\operatorname{cg} 18823846$ & PRKG1 & 0.000903 & Higher & $-3.95 /-4.20$ \\
\hline cg25405123 & RYR3 & 0.000427 & Higher & $-3.61 /-3.89$ \\
\hline
\end{tabular}

a 'Lower' indicates that methylation at the CpG site is lower in cases vs controls and 'higher' means higher methylation in cases vs controls.

b M-values indicate normalized methylation values. 
Table 3

Linear regression results between long-term depression pathway methylation sites and mood, vigilance and tiredness, and sleep.

\begin{tabular}{|c|c|c|c|c|c|c|c|c|c|c|}
\hline \multirow[t]{3}{*}{ Locus (gene) } & \multirow{2}{*}{\multicolumn{2}{|c|}{$\begin{array}{l}\text { Mood } \\
\text { BDI-19 }\end{array}$}} & \multirow{2}{*}{\multicolumn{2}{|c|}{$\begin{array}{l}\text { Vigilance and } \\
\text { tiredness } \\
\text { Median RT }\end{array}$}} & \multicolumn{6}{|l|}{ Sleep } \\
\hline & & & & & \multicolumn{2}{|l|}{ PDSS } & \multicolumn{2}{|l|}{ AIS } & \multicolumn{2}{|c|}{ SWAdiss } \\
\hline & B & $p$ & $\mathrm{~B}$ & $p$ & B & $p$ & $\mathrm{~B}$ & $p$ & $\mathrm{~B}$ & $p$ \\
\hline $\operatorname{cg} 1684176$ (CACNG1) & -0.69 & 0.002 & -0.44 & 0.08 & -0.58 & 0.02 & -0.68 & 0.003 & 0.38 & 0.14 \\
\hline cg22025854 (CACNG6) & 0.44 & 0.08 & 0.40 & 0.11 & 0.19 & 0.49 & 0.55 & 0.02 & -0.25 & 0.36 \\
\hline $\operatorname{cg} 08364956$ (GRM6) & -0.47 & 0.06 & -0.26 & 0.31 & -0.60 & 0.01 & -0.75 & 0.001 & 0.38 & 0.15 \\
\hline cg05110803 (IGF1R) & -0.50 & 0.04 & -0.28 & 0.29 & -0.79 & $0.00029^{\mathrm{a}, \mathrm{b}}$ & -0.68 & 0.003 & 0.24 & 0.38 \\
\hline $\operatorname{cg} 19161850(E R K ~ 12)$ & 0.55 & 0.023 & 0.21 & 0.43 & 0.72 & $0.002^{\mathrm{a}}$ & 0.65 & 0.005 & -0.47 & 0.67 \\
\hline cg12066398 (PLA2G16) & 0.77 & $0.00031^{\mathrm{b}, \mathrm{a}}$ & 0.66 & 0.004 & 0.33 & 0.21 & 0.70 & 0.002 & -0.32 & 0.23 \\
\hline $\operatorname{cg} 04367351(P L A 2 R 1)$ & 0.48 & 0.053 & 0.17 & 0.51 & 0.77 & $0.00047^{\mathrm{a}, \mathrm{b}}$ & 0.68 & 0.003 & -0.30 & 0.26 \\
\hline cg02263165 (PPP2R5C) & 0.64 & 0.006 & 0.51 & 0.035 & 0.32 & 0.23 & 0.65 & 0.005 & -0.67 & $0.004^{\mathrm{a}}$ \\
\hline cg18823846 (PRKG1) & 0.57 & 0.018 & 0.49 & 0.045 & 0.50 & 0.051 & 0.60 & 0.010 & -0.16 & 0.56 \\
\hline cg25405123 (RYR) & 0.64 & 0.006 & 0.42 & 0.095 & 0.44 & 0.091 & 0.76 & $0.00041^{\mathrm{b}}$ & -0.54 & 0.031 \\
\hline
\end{tabular}

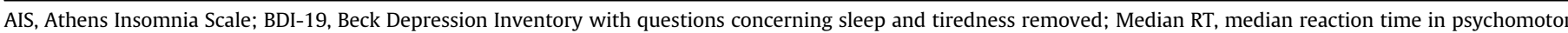
vigilance task (PVT); PDSS, Pediatric Sleepiness Scale; SWAdiss, slow wave dissipation during first episode of the night measured from frontal electrodes.

a Association is significant at $<0.05$ level when case-control status is controlled for (nominal).

b Association is significant after Bonferroni-correction.

correlations of $p<0.05$ (not corrected for multiple testing) were observed for $\operatorname{cg} 05110803$ (from IGF1R), $\operatorname{cg} 19161850$ (ERK 12) and cg04367351 (PLA2R1) to subjective tiredness ( $p=0.007,0.041$ and 0.011 , respectively), cg12066398 (PLA2G16) to depressed mood $(p=0.031)$ and $c g 02263165(P P P 2 R 5 C)$ to slower SWA dissipation $(p=0.02)$ (Table 3, Fig. 2(a)-(e)).

Although the statistical significance was limited due to small sample size, these findings make biologic sense, with similar trends observed for both cases and controls. For example, lower methylation levels at cg05110803 (IGF1R), which showed relative hypomethylation in cases compared to controls in the initial case-control analysis, also associated with more pronounced tiredness in both cases and controls (Fig. 2(a)). Similarly, higher methylation levels at cg02263165 (PPP2R5C), which showed relative hypermethylation in cases in the initial analysis, associated with slower SWA dissipation among both cases and controls (Fig. 2(c)).

\section{Discussion}

In this study we searched for deviating methylation patterns in adolescents with depressive disorder and sleep difficulties, as compared to age- and gender-matched controls. Due to small sample size, this was aimed as a hypothesis-generating rather than hypothesis-confirming study. However, we found enrichment of DMPs at genes from a canonical pathway essential for learning and memory consolidation, the synaptic LTD pathway. This finding adds to the systematic evidence of the connection between depressive disorder, sleep and cognition, and supports earlier results concerning the role of sleep in synaptic plasticity, especially during brain development [46].

LTD acts in memory consolidation and synaptic plasticity together with long-term potentiation. LTD can deactivate memories that are activated by LTP and, thus, learning can be seen as a result of a balance between these two processes [47]. LTD also plays a crucial role in the formation of new memories [48]. Sleep deprivation can significantly increase LTD in rat hippocampus [49], and the possible mechanism could be related to an altered expression of $\gamma$-aminobutyric acid (GABA) B receptors [50].

It is postulated that the decrease in SWA during the course of sleep is associated with reduced synaptic strength and is thus related to memory consolidation [51]. Our previous results on the same sample of adolescents indicated that this SWA dissipation that occurs normally during healthy sleep is affected by depression and related sleep disturbances [23]. The results of the current study further propose a link between SWA dissipation and changes in methylation of the Protein Phosphatase 2 regulatory subunit B'gamma (PPP2RC5).

According to an epigenome consortium [52], certain tissues have common methylation patterns across each other, while others do not. With the Epigenome browser (http://epigenomegateway. wustl.edu/browser/) (WashU), we explored methylation values and expression levels of PPP2R5C's cg12066398, located at chromosome 14; 102,276,300. It appeared that methylation levels of different histone methylation arrays were comparable between peripheral blood leukocytes and brain hippocampal cells, but no data concerning $\mathrm{CpG}$ methylation was available from brain tissue and peripheral blood leukocytes for comparison.

PPP2R5C belongs to the phosphatase $2 \mathrm{~A}$ regulatory subunit $\mathrm{B}$ family. This phosphatizing enzyme is implicated in the negative control of cell growth and division (RefSeq, Jul 2008). Mutations in this enzyme family are related to human overgrowth syndromes accompanied by intellectual disabilities [53]. This family of enzymes is highly and widely expressed in the human brain [54]. Protein phosphatases are responsible for the induction of LTD and initiating the cascade with a slow $\mathrm{Ca}^{+}$ion flow into neurons $[55,56]$. We found that those patients with the highest levels of methylation in the promotor region of PPP2R5C, suggesting slower LTD induction, also had the flattest SWA dissipation pattern throughout the night. The association between methylation and gene expression can either be active, where methylation directly affects gene expression, or passive, meaning that methylation is just a byproduct of expression regulation. Either way, it can be regarded as an indicator of gene expression regulation [57,58]. It has also been shown that even small changes in DNA methylation may result in marked differences in phenotype [59].

The other genes of the LTD pathway found to correlate with sleep and depression in the current study were ERK12, PLA2G16, IG1FR and PLA2R1. Extracellular signal-regulated kinase (ERK) is a group of protein kinases that belong to a larger family of mitogenactivated protein kinases (MAPK). ERK12 is related to the formation of LTP and is partially regulated by phosphatases, such as PPP2R5C $[60,61]$. Notably, we found correlation between daytime sleepiness and ERK12, which is in line with findings that this enzyme is involved in the daytime enhancement of LTP [62]. There is evidence that for synaptic plasticity, LTP is favoured during the daytime and contributes to the strengthening of synapses, whereas LTD is induced by slow wave sleep (SWS) [62]. This creates a balance 
a

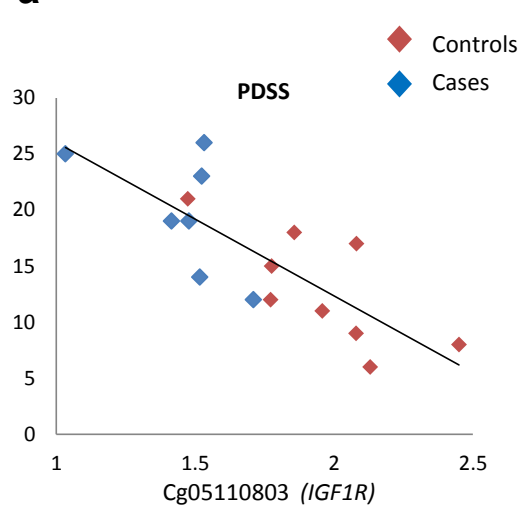

b

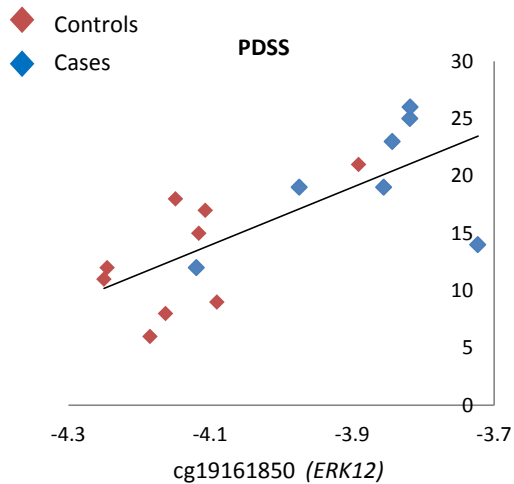

C

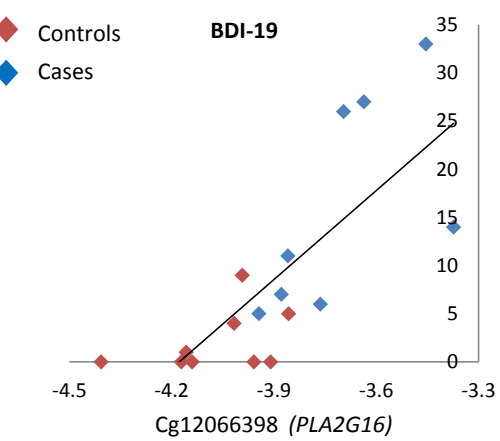

d

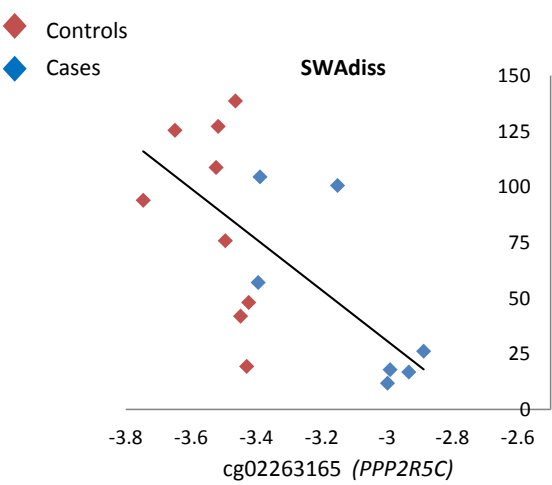

e

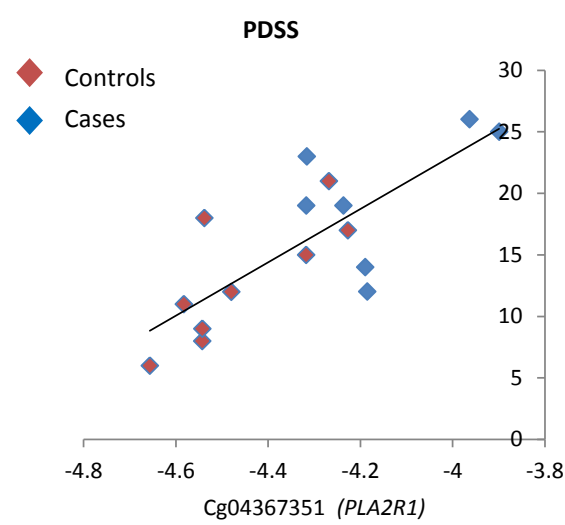

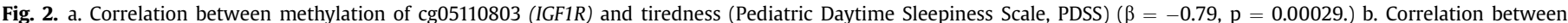

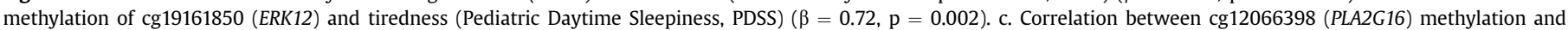

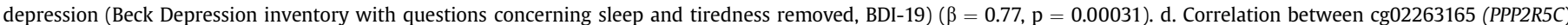

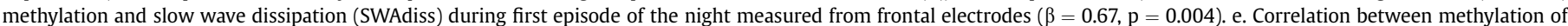
cg04367351 (PLA2R1) and tiredness (Pediatric Daytime Sleepiness, PDSS) $(\beta=0.77, \mathrm{p}=0.00047$ )

called synaptic homeostasis between synaptic strengthening and weakening, allowing new information to be consolidated while preserving previous information. PLA2G16 (Phospholipase A2 Group XVI) mediates the ERK12 pathway [63]. IGF1R (Insulin-like Growth Factor 1 Receptor) is a protein coding gene that plays a crucial role in central nervous system (CNS) development, maturation and neuronal plasticity [64]. Blocking IGF1R in mouse Purkinje cells leads to cessation of LTD [65]. PLA2R1 (Phospholipase A2 Receptor 1 ) is a protein-coding gene that has a variety of roles depending on tissue. Its role in LTD is somewhat unclear, but it is identified as a part of both pre- and postsynaptic cascades related to regulation of LTD and is activated by ERK 12. Neuronal activity during SWS has been shown to promote LTD in neurons [47,51], and the slow oscillation that happens in neurons during SWS favours LTD over LTP [48], suggesting that the SWS stage is strongly related to LTD. In contrast, other sleep stages, especially REM sleep, have a stronger effect on LTP [56]. Different sleep disturbances can therefore affect LTP and LTD differently [56,66]. Since in our sample there was no marked difference in REM sleep between cases and controls [23], it is plausible that there were no differences in LTP genetic pathways either.

We did not find marked differences in cognitive performance between cases and controls (unpublished data), except for a longer mean reaction time, minimum reaction time, and fastest $10 \%$ reaction time in cases vs controls, implying lower vigilance among cases. This is in line with previous studies concerning the effect of 
depression on adolescent cognitive performance [65] and the effect of sleep difficulties on cognition [67]. This is somewhat in contrast to findings in adults, however, putatively reflecting the specific developmental stage involving marked changes in the reorganizing of neural networks (synaptic pruning) and consolidation of cortical neural layers $[68,69]$. Given the variable but, on average, quite long duration of depressive disorder (mean length from beginning of episode to sampling: $415 \pm 377$ weeks), the cases in our sample may have been able to compensate for the effect of depression and sleep disturbance on cognition. Although the changes in methylation of the LTD pathway would suggest compromised synaptic plasticity, it is thus possible that altered epigenetic regulation of the LTD pathway is a compensatory mechanism for changes in SWA dissipation, acting to protect individuals from memory and other cognitive impairments during adolescence.

The strengths of the study include the carefully selected and homogeneous sample: all participants were boys, they were all non-medicated, the age range of the sample was narrow, and the participants underwent careful clinical assessment. Further, the use of polysomnography to objectively assess sleep is also one of the major strengths of the study. An evident shortcoming in our study includes the small sample size. Because of this, we were unable to conduct reliable analyses for possible methylation quantitative loci (mQTLs). mQTLs are SNPs that can have an effect on the methylation of one or several distant $\mathrm{CpG}$ sites. Thus, the results need to be duplicated and extended in larger samples before more extensive interpretation.

We did not adjust analyses for depression length but, based on our analyses, that would lead to exploration of cases only and thus an even more limited sample size. All participants remained free from psychotropic and other medication during the study period. The patients received, however, 'treatment as usual' at the Helsinki University Hospital adolescent psychiatric outpatient unit, which usually consists of individual (frequency typically once a week) and family appointments (frequency typically once a month) and thus we cannot rule out the effect of psychosocial treatment on the findings from the secondary analysis. We analysed epigenetic alterations from peripheral blood leukocytes, and it is known that many epigenetic alterations are tissue specific [52]. It is not, however, an easy task to obtain living brain tissue for epigenetic studies. Peripheral blood leukocytes serve as a good option to study sleeprelated changes in the CNS since the brain's lymphatic drainage system connects the immune system, CNS and sleep tightly together [70]. Therefore, it is exciting that we found an association between sleep and methylation of the LTD pathway. Some databases exist where the methylation level of peripheral blood leukocytes and brain tissues can be found (eg, Epigenome browser), but they do not cover all sites or all methylation arrays. Moreover, comparable samples from the same individual, drawn at the same time point, do not exist, making comparison even more uncertain [71]. Further, only boys were included in our sample, limiting the generalizability of the findings to girls.

Our Pathway analysis did not correct for multiple testing, but correction would not change which pathways arise as most likely to associate with a set of genes derived from DMPs. This approach allows the generation of a new hypothesis concerning the interplay between sleep, depression and cognition.

In conclusion, our work presents new insights into the systematic effects of disordered sleep and depression, resulting in a deviating pattern of DNA methylation in blood leukocytes and providing evidence of compromised synaptic plasticity among a group of adolescents with depressive disorder and disturbed sleep. However, further studies involving more detailed neurobiological analysis in both experimental and naturalistic settings should be conducted in order to further explore the link between systematic and brain-specific epigenetic modifications of LTD pathway genes and their relationships with depression and disordered sleep.

\section{Acknowledgements}

Financial support for the study includes a special federal grant (TYH 2013342) to T.P. and funding from the Academy of Finland (grant number 276612 to A.S.U. and number 290039 to T.P.), Emil Aaltonen Foundation, Finnish Medical Foundation, Finnish Brain Foundation, Orion-Farmos Research Foundation, Päivikki and Sakari Sohlberg Foundation, and the Foundation for Psychocultural Research. We also wish to thank Auli Toivola for her valuable contribution regarding laboratory work.

\section{Conflict of interest}

None of the authors reported conflict of interest.

The ICMJE Uniform Disclosure Form for Potential Conflicts of Interest associated with this article can be viewed by clicking on the following link: https://doi.org/10.1016/j.sleep.2019.01.050.

\section{Appendix A. Supplementary data}

Supplementary data to this article can be found online at https://doi.org/10.1016/j.sleep.2019.01.050.

\section{References}

[1] Urrila AS, Paunio T, Palomaki E, et al. Sleep in adolescent depression: physiological perspectives. Acta Physiol 2015;213(4):758-77.

[2] Blake MJ, Trinder JA, Allen NB. Mechanisms underlying the association between insomnia, anxiety, and depression in adolescence: implications for behavioral sleep interventions. Clin Psychol Rev 2018;63:25-40.

[3] Lovato N, Gradisar M. A meta-analysis and model of the relationship between sleep and depression in adolescents: recommendations for future research and clinical practice. Sleep Med Rev 2014;18(6):521-9.

[4] Palagini L, Baglioni C, Ciapparelli A, et al. REM sleep dysregulation in depression: state of the art. Sleep Med Rev 2013:17(5):377-90.

[5] Dickinson DL, Wolkow AP, Rajaratnam SMW, et al. Personal sleep debt and daytime sleepiness mediate the relationship between sleep and mental health outcomes in young adults. Depress Anxiety 2018;35(8):775-83.

[6] American Psychiatric Association. Diagnostic and statistical manual of mental disorders. 5th ed. 2013. Washington, DC.

[7] Parkinson GT, Hanley JG. Mechanisms of AMPA receptor endosomal sorting. Front Mol Neurosci 2018;11:440.

[8] Raven F, Van der Zee EA, Meerlo P, et al. The role of sleep in regulating structural plasticity and synaptic strength: implications for memory and cognitive function. Sleep Med Rev 2018;39:3-11.

[9] Tononi G, Cirelli C. Sleep and the price of plasticity: from synaptic and cellular homeostasis to memory consolidation and integration. Neuron 2014;81(1): $12-34$.

[10] Wolf E, Kuhn M, Normann C, et al. Synaptic plasticity model of therapeutic sleep deprivation in major depression. Sleep Med Rev 2016;30:53-62.

[11] Gouin JP, Connors J, Kiecolt-Glaser JK, et al. Altered expression of circadian rhythm genes among individuals with a history of depression. J Affect Disord 2010:126(1-2):161-6.

[12] Moller-Levet CS, Archer SN, Bucca G, et al. Effects of insufficient sleep on circadian rhythmicity and expression amplitude of the human blood transcriptome. Proc Natl Acad Sci U S A 2013;110(12):E1132-41.

[13] Luppino FS, de Wit LM, Bouvy PF, et al. Overweight, obesity, and depression: systematic review and meta-analysis of longitudinal studies. Arch Gen Psychiatry 2010;67(3):220-9.

[14] Okubo N, Matsuzaka M, Takahashi I, et al. Relationship between self-reported sleep quality and metabolic syndrome in general population. BMC Public Health 2014; $14: 562$.

[15] Kessler RC. The effects of stressful life events on depression. Annu Rev Psychol 1997;48:191-214.

[16] Gudsnuk K, Champagne FA. Epigenetic influence of stress and the socia environment. ILAR J 2012;53(3-4):279-88.

[17] Gavrieli A, Farr OM, Davis CR, et al. Early life adversity and/or posttraumatic stress disorder severity are associated with poor diet quality, including consumption of trans fatty acids, and fewer hours of resting or sleeping in a US middle-aged population: a cross-sectional and prospective study. Metabolism 2015;64(11):1597-610.

[18] Menke A, Binder EB. Epigenetic alterations in depression and antidepressant treatment. Dialogues Clin Neurosci 2014;16(3):395-404. 
[19] Hobara T, Uchida S, Otsuki K, et al. Altered gene expression of histone deacetylases in mood disorder patients. J Psychiatr Res 2010;44(5):263-70.

[20] Massart R, Freyburger M, Suderman M, et al. The genome-wide landscape of DNA methylation and hydroxymethylation in response to sleep deprivation impacts on synaptic plasticity genes. Transl Psychiatry 2014;4:e347.

[21] Maag JL, Kaczorowski DC, Panja D, et al. Widespread promoter methylation of synaptic plasticity genes in long-term potentiation in the adult brain in vivo. BMC Genom 2017;18(1):250.

[22] Carroll JE, Irwin MR, Levine M, et al. Epigenetic aging and immune senescence in women with insomnia symptoms: findings from the women's health initiative study. Biol Psychiatry 2017;81(2):136-44.

[23] Santangeli O, Porkka-Heiskanen T, Virkkala J, et al. Sleep and slow-wave activity in depressed adolescent boys: a preliminary study. Sleep Med 2017;38: 24-30.

[24] Urrila AS, Hakkarainen A, Castaneda A, et al. Frontal cortex myo-inositol is associated with sleep and depression in adolescents: a proton magnetic resonance spectroscopy study. Neuropsychobiology 2017;75(1):21-31.

[25] Merikanto I, Partonen T, Paunio T, et al. Advanced phases and reduced amplitudes are suggested to characterize the daily rest-activity cycles in depressed adolescent boys. Chronobiol Int 2017;34(7):967-76.

[26] Kaufman J, Birmaher B, Brent D, et al. Schedule for affective disorders and schizophrenia for school-age children-present and lifetime version (K-SADSPL): initial reliability and validity data. J Am Acad Child Adolesc Psychiatry 1997;36(7):980-8.

[27] Wentz E, Gillberg IC, Anckarsater H, et al. Reproduction and offspring status 18 years after teenage-onset anorexia nervosa-a controlled communitybased study. Int J Eat Disord 2009;42(6):483-91.

[28] Ulberg R, Hersoug AG, Hoglend P. Treatment of adolescents with depression: the effect of transference interventions in a randomized controlled study of dynamic psychotherapy. Trials 2012;13:159.

[29] Beck AT, Ward CH, Mendelson M, et al. An inventory for measuring depression. Arch Gen Psychiatry 1961;4:561-71

[30] Marton P, Churchard M, Kutcher S, et al. Diagnostic utility of the Beck Depression Inventory with adolescent psychiatric outpatients and inpatients. Can J Psychiatry 1991;36(6):428-31.

[31] Brooks SJ, Kutcher S. Diagnosis and measurement of adolescent depression: a review of commonly utilized instruments. J Child Adolesc Psychopharmacol 2001;11(4):341-76.

[32] Basner M, Hermosillo E, Nasrini J, et al. Repeated administration effects on psychomotor vigilance test (PVT) performance. Sleep 2017;41(1). https:// doi.org/10.1093/sleep/zsx187.

[33] Urrila AS, Karlsson L, Kiviruusu O, et al. Sleep complaints among adolescent outpatients with major depressive disorder. Sleep Med 2012;13(7):816-23.

[34] Soldatos CR, Dikeos DG, Paparrigopoulos TJ. Athens Insomnia Scale: validation of an instrument based on ICD-10 criteria. J Psychosom Res 2000;48(6): 555-60.

[35] Drake C, Nickel C, Burduvali E, et al. The pediatric daytime sleepiness scale (PDSS): sleep habits and school outcomes in middle-school children. Sleep 2003;26(4):455-8.

[36] Aryee MJ, Jaffe AE, Corrada-Bravo H, et al. Minfi: a flexible and comprehensive bioconductor package for the analysis of infinium DNA methylation microarrays. Bioinformatics 2014;30(10):1363-9.

[37] Dedeurwaerder S, Defrance M, Calonne E, et al. Evaluation of the infinium methylation 450K technology. Epigenomics 2011;3(6):771-84.

[38] Bibikova M, Barnes B, Tsan C, et al. High density DNA methylation array with single CpG site resolution. Genomics 2011;98(4):288-95.

[39] Houseman EA, Accomando WP, Koestler DC, et al. DNA methylation arrays as surrogate measures of cell mixture distribution. BMC Bioinf 2012:13:86.

[40] Reinius LE, Acevedo N, Joerink M, et al. Differential DNA methylation in purified human blood cells: implications for cell lineage and studies on disease susceptibility. PLoS One 2012;7(7):e41361.

[41] Maksimovic J, Gordon L, Oshlack ASWAN. Subset-quantile within array normalization for illumina infinium HumanMethylation450 BeadChips. Genome Biol 2012;13(6):R44.

[42] Smyth GK. Linear models and empirical bayes methods for assessing differential expression in microarray experiments. Stat Appl Genet Mol Biol 2004;3. Article3.

[43] Benjamini Y, Yosef $\mathrm{H}$. Controlling the false discovery rate: a practical and powerful approach to multiple testing. J R Stat Soc Ser B Stat Methodol 1995;57(1):289-300.

[44] Nalesso G, Sherwood J, Bertrand J, et al. WNT-3A modulates articular chondrocyte phenotype by activating both canonical and noncanonical pathways. J Cell Biol 2011;193(3):551-64.
[45] Talbot LS, McGlinchey EL, Kaplan KA, et al. Sleep deprivation in adolescents and adults: changes in affect. Emotion 2010;10(6):831-41.

[46] Lo JC, Groeger JA, Santhi N, et al. Effects of partial and acute total sleep deprivation on performance across cognitive domains, individuals and circadian phase. PLoS One 2012;7(9):e45987.

[47] Vyazovskiy VV, Cirelli C, Pfister-Genskow M, et al. Molecular and electrophysiological evidence for net synaptic potentiation in wake and depression in sleep. Nat Neurosci 2008;11(2):200-8.

[48] Nabavi S, Fox R, Proulx CD, et al. Engineering a memory with LTD and LTP. Nature 2014;511(7509):348-52.

[49] Connor SA, Wang YT. A place at the table: LTD as a mediator of memory genesis. Neuroscientist 2016;22(4):359-71.

[50] Tadavarty R, Rajput PS, Wong JM, et al. Sleep-deprivation induces changes in $\mathrm{GABA}(\mathrm{B})$ and $\mathrm{mGlu}$ receptor expression and has consequences for synaptic long-term depression. PLoS One 2011;6(9):e24933.

[51] Tononi G. Slow wave homeostasis and synaptic plasticity. J Clin Sleep Med 2009;5(2 Suppl):S16-9.

[52] Roadmap Epigenomics C, Kundaje A, Meuleman W, et al. Integrative analysis of 111 reference human epigenomes. Nature 2015;518(7539):317-30.

[53] Loveday C, Tatton-Brown K, Clarke M, et al. Mutations in the PP2A regulatory subunit B family genes PPP2R5B, PPP2R5C and PPP2R5D cause human overgrowth. Hum Mol Genet 2015;24(17):4775-9.

[54] McCright B, Brothman AR, Virshup DM. Assignment of human protein phosphatase $2 \mathrm{~A}$ regulatory subunit genes b56alpha, b56beta, b56gamma, b56delta, and b56epsilon (PPP2R5A-PPP2R5E), highly expressed in muscle and brain, to chromosome regions 1q41, 11q12, 3p21, 6p21.1, and 7p11.2-> p12. Genomics 1996;36(1):168-70.

[55] Benington JH, Frank MG. Cellular and molecular connections between sleep and synaptic plasticity. Prog Neurobiol 2003;69(2):71-101.

[56] Belmeguenai A, Hansel C. A role for protein phosphatases 1,2A, and 2B in cerebellar long-term potentiation. J Neurosci 2005;25(46):10768-72.

[57] Gutierrez-Arcelus M, Lappalainen T, Montgomery SB, et al. Passive and active DNA methylation and the interplay with genetic variation in gene regulation. Elife 2013;2:e0523.

[58] Guo S, Diep D, Plongthongkum N, et al. Identification of methylation haplotype blocks aids in deconvolution of heterogeneous tissue samples and tumor tissue-of-origin mapping from plasma DNA. Nat Genet 2017;49(4):635-42.

[59] Leenen FA, Muller CP, Turner JD. DNA methylation: conducting the orchestra from exposure to phenotype? Clin Epigenet 2016;8:92.

[60] Guan Z, Peng X, Fang J. Sleep deprivation impairs spatial memory and decreases extracellular signal-regulated kinase phosphorylation in the hippocampus. Brain Res 2004;1018(1):38-47.

[61] Ito-Ishida A, Kakegawa W, Yuzaki M. ERK1/2 but not p38 MAP kinase is essential for the long-term depression in mouse cerebellar slices. Eur J Neurosci 2006;24(6):1617-22.

[62] Yang Z, Zhang W, Wang M, et al. Effects of daytime, night and sleep pressure on long-term depression in the hippocampus in vivo. Neurosci Lett 2012:511(2):106-9.

[63] Li L, Liang S, Wasylishen AR, et al. PLA2G16 promotes osteosarcoma metastasis and drug resistance via the MAPK pathway. Oncotarget 2016;7(14): 18021-35.

[64] Dyer AH, Vahdatpour C, Sanfeliu A, et al. The role of Insulin-Like Growth Factor 1 (IGF-1) in brain development, maturation and neuroplasticity. Neuroscience 2016;325:89-99.

[65] Benitez A Gunstad J. Poor sleep quality diminishes cognitive functioning independent of depression and anxiety in healthy young adults. Clin Neuropsychol 2012;26(2):214-23.

[66] Diekelmann S, Born J. The memory function of sleep. Nat Rev Neurosci 2010;11(2):114-26.

[67] Blakemore SJ. Imaging brain development: the adolescent brain. Neuroimage 2012;61(2):397-406.

[68] Tamnes CK, Herting MM, Goddings AL, et al. Development of the cerebral cortex across adolescence: a multisample study of inter-related longitudinal changes in cortical volume, surface area, and thickness. J Neurosci 2017;37(12):3402-12.

[69] Nebes RD, Buysse DJ, Halligan EM, et al. Self-reported sleep quality predicts poor cognitive performance in healthy older adults. J Gerontol B Psychol Sci Soc Sci 2009;64(2):180-7.

[70] Sun BL, Wang LH, Yang T, et al. Lymphatic drainage system of the brain: a novel target for intervention of neurological diseases. Prog Neurobiol 2018;163-164:118-43.

[71] Tejedor JR, Fraga MF. Interindividual epigenetic variability: sound or noise? Bioessays 2017;39(7). https://doi.org/10.1002/bies.201700055. 